\title{
Hydrodynamic hull form optimization of fast catamarans using surrogate models
}

\author{
Mittendorf, Malte; Papanikolaou, Apostolos D.
}

Published in:

Ship Technology Research

Link to article, DOI:

10.1080/09377255.2020.1802165

Publication date:

2021

Document Version

Peer reviewed version

Link back to DTU Orbit

Citation $(A P A)$ :

Mittendorf, M., \& Papanikolaou, A. D. (2021). Hydrodynamic hull form optimization of fast catamarans using surrogate models. Ship Technology Research, 68(1), 14-26 . https://doi.org/10.1080/09377255.2020.1802165

\section{General rights}

Copyright and moral rights for the publications made accessible in the public portal are retained by the authors and/or other copyright owners and it is a condition of accessing publications that users recognise and abide by the legal requirements associated with these rights.

- Users may download and print one copy of any publication from the public portal for the purpose of private study or research.

- You may not further distribute the material or use it for any profit-making activity or commercial gain

- You may freely distribute the URL identifying the publication in the public portal 


\title{
Hydrodynamic Hull Form Optimisation of Fast Catamarans Using Surrogate Models
}

\author{
Malte Mittendorf ${ }^{1,2}$ and Apostolos D. Papanikolaou ${ }^{3,4, *}$ \\ ${ }^{1}$ Technical University of Denmark (DTU), Department of Mechanical Engineering \\ Nils Koppels Allé 403, 2800 Kgs. Lyngby, Denmark \\ ${ }^{2}$ (former) University of Applied Sciences Kiel (UAS), Institute of Naval Architecture \\ Sokratesplatz 1, 24149 Kiel, Germany \\ ${ }^{3}$ Hamburg Ship Model Basin (HSVA), Computational Fluid Dynamics Department \\ Bramfelder Straße 164, 22305 Hamburg, Germany \\ ${ }^{4}$ National Technical University of Athens (NTUA), Ship Design Laboratory \\ Heroon Polytechniou 9, Zografou - 15773 Athens, Greece \\ *Corresponding author, papa@ deslab.ntua.gr
}

\begin{abstract}
This paper demonstrates the practical benefit of using efficient computational methods to optimise fast catamarans hydrodynamically. The development of a simplified panel method, which is based on thin ship theory, and the validation of the associated code for the prediction of the calm water resistance of twin-hull vessels is presented. The method was applied in the multi-objective optimisation of a fast, zero-emission, battery-driven catamaran by a genetic algorithm, while considering the ensuing design constraints, such as battery weight and space requirements. Results of the study were compared with resistance predictions from an advanced, non-linear Rankine panel method. Moreover, surrogate models for the approximation of the individual objective functions were implemented to speed up the optimisation process involving several hundred parametrically generated designs. Obtained results were also validated against results of a viscous CFD solver and showed great potential for the use of the proposed modified thin ship theory in connection with empirical corrections in the field of resistance prediction and hull form optimisation of fast catamarans and of slender hulls in general.
\end{abstract}

\section{KEYWORDS}

Hydrodynamic Optimisation; Thin Ship Theory Panel Method, Fast Zero-Emission Catamarans, Surrogate Modelling; Genetic Algorithm;

\section{INTRODUCTION}

The limited availability of fossil fuels and the increased environmental awareness by society have forced national and international policy makers as well as the IMO (International Maritime Organization) to promote and even legislate the use of alternative, renewable and environmentally friendly energy sources by the maritime industry in the next few decades. In this respect, the development of innovative, zero-emission ships for coastal and deep-sea operation is a far-reaching goal for researchers and naval architects in the years to come. This goal is also advanced by the EU funded research project TrAM (Transport: Advanced and Modular), which aims to design and manufacture a zero-emission, fast catamaran passenger ship for coastal operation (TrAM, 2018-2022). The present case study is dealing with the hydrodynamic optimisation of a fully batterydriven fast catamaran vessel and adopts a modular design and construction practice; thus, it will be at the forefront of related maritime industry developments. Due to its innovative nature, the application of virtual prototyping in this vessel's design is imperative. In this respect, the paper pursues a simulation-driven global 
hull form optimisation approach for the "TrAM Stavanger demonstrator", an artistic sketch of which can be seen in Fig. 1. For general information about the adopted design optimisation methodologies, see Nowacki (2003), Papanikolaou (2010) and Bertram (2017).

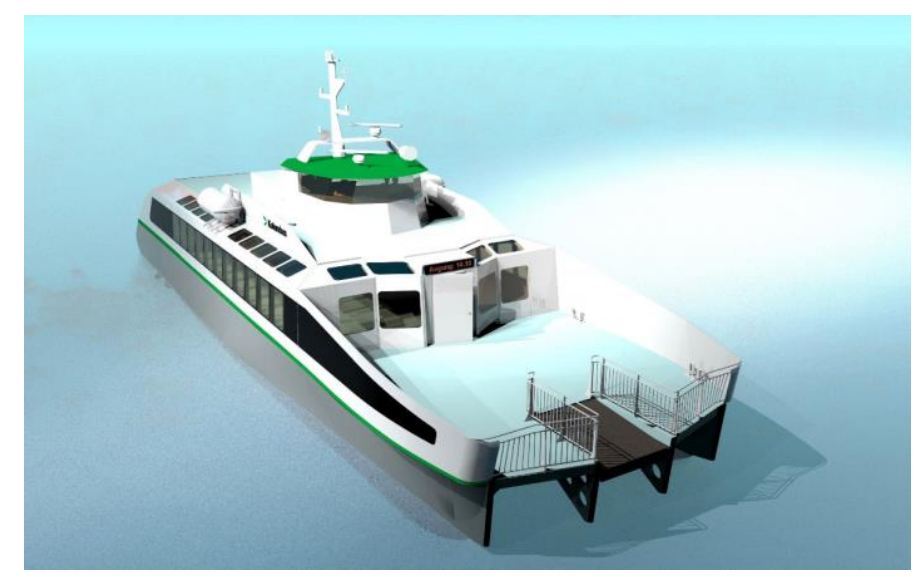

Figure 1: Artist Impression of the TrAM Stavanger Demonstrator (TrAM, 2018-2022).

The preliminary operational profile of the case study vessel that will be operating in a waterborne urban bus service, is predominantly characterised by the strong variation of its service speed and frequent acceleration/deceleration phases; hence, the definition of one single design speed for optimisation is not possible. Instead, multiple speeds in the range of $0.7 \leq F n \leq 0.9$ are considered as objective functions leading to a multi-objective optimisation problem. The multi-objective optimisation within the ship design process is one of the pillars of the EU funded HOLISHIP project, which is forming the basis of the herein presented work (HOLISHIP 2016-2020). The PIDO (Process Integration and Design Optimization) software CAESES ${ }^{\circledR}$ (Computer Aided Engineering System Empowering Simulation) by FRIENDSHIP SYSTEMS is the main design integration platform of the HOLISHIP project that is also used herein, Harries and Abt (2019).

Catamarans are superior to monohulls with the same displacement in terms of transverse stability and exploitable deck area, but they have generally an up to $35 \%$ larger wetted surface area, leading to increased frictional resistance; this drawback has to be compensated, to some extent, by lower wave resistance. The wave resistance of a catamaran for a certain speed is generally minimised by proper distribution of its displacement, which is inherently reduced by the slender demihulls and by proper separation distance. This also allows the application of the linear slender or thin ship theory, as shown by Papanikolaou and Androulakakis (1991) in their Lagrange multiplicator optimisation procedure for fast SWATH (Small Waterplane Area Twinhull) ships. Danisman et al. (2002) use thin ship theory in the frame of a wave resistance optimisation of a catamaran's aft ship in medium speed range by combining it with quadratic programming. Their focal point, however, is the optimisation by an artificial neural network (ANN) being trained on results of a Rankine panel method. This approach is considered as surrogate modelling and saves computational effort by approximating the associated objective function. Zaraphonitis et al. (2003) present a multi-objective hull form optimisation regarding not only the calm water resistance, but also a wave wash criterion. The optimisation is carried out with a genetic algorithm applied to a Rankine panel method for both a monohull and a catamaran topology. Bertram et al. (2017) provide a simulation-driven optimisation of a conceptual trimaran design. In doing so, not only the operational profile is taken into account, but also the seakeeping attitude. The design exploration is performed by a Rankine panel method and a RANSE (Reynolds-averaged Navier Stokes Equations) solver then validates certain generated designs.

The aim of the present paper is to demonstrate the practical benefit by the use of a simplified thin ship theory panel method in conjunction with an empirical correction for the stern viscous flow. The proposed method can be readily used in the early design stages of fast catamarans and achieves comparable accuracy to an advanced non-linear Rankine panel method, but with considerably less computational effort. The use of surrogate models approximating the examined objective functions is another key element of the applied methodology, which leads to a further reduction of the computational effort. 


\section{THEORETICAL BACKGROUND}

The assumption of an irrotational, homogenous and incompressible flow allows the description of the flow by a velocity potential $\Phi$ fulfilling Laplace Equation $\Delta \Phi=0$. Thus, we consider a steady, potential flow caused by a ship moving with constant forward speed $U_{\infty}$ in a non-bounded waterway on a free surface. A right-handed Cartesian coordinate system $0-x-y-z$, fixed to the moving ship is introduced, with the $0-x-y$ plane on the calm free water surface, the 0 -x-z plane on ship's centerplane; the $x$-axis coincides with the direction of motion, whereas the z-axis shows upwards. In the absence of viscosity, the ship experiences only a wave resistance due to the lost energy by the generation of surface waves. It is well established that the wave resistance $R_{W}$ of thin ships $(B / L<<1)$ may be determined by the Fourier integral method of Michell (1898). The boundary value problem is addressed by assuming a distribution of Kelvin sources on the symmetry plane of the hull. According to thin ship theory the source densities $\sigma$ are dependent on the free stream velocity $U_{\infty}$ and the local longitudinal slope of the waterlines $f$.

$$
\sigma(x, 0, z)=-\frac{U_{\infty}}{2 \pi} \frac{\partial f}{\partial x}(x, z)
$$

The wave resistance force is then expressed by the sum of the products of the local source densities and the ship's speed of advance (Lagally theorem, 1922). An elaboration of the theoretical background of thin ship theory by Michell one may find in Faltinsen (2005). For the calculation of the wave resistance of a twin-hull arrangement (catamaran) we employ an extension of Michell's thin ship theory; namely, a formulation of Srettensky (1936) for the resistance of a thin ship moving in a canal of width $W$, as modified by Chapman (1972) for the wave resistance of twin-hull vessels. Moreover, we adopt an additional deviation from the Michell thin ship theory with the placement of the assumed singularities on the demihulls' wetted surface being discretised by panels, as originally proposed by Hess and Smith (1967), instead of on their centerplane, Papanikolaou and Androulakakis (1991). This is justified by the fact that when solving the associated boundary value problem by Green's function method, leading to the solution of a set of integral equations for the unknown source strengths, the asymptotic expansion of the solution for the source density is equal to Michell's solution (Kaklis and Papanikolaou, 1992). The immersed part of one-half of the demihull is defined by $n_{\text {pan }}$ number of panels having a triangular or quadrilateral shape and the wave resistance is calculated by Eq. 2.

$$
R_{W}=-16 \pi \rho k_{0} W^{-1}\left(\frac{U_{\infty}}{2 \pi}\right)^{2} \sum_{n=0}^{\infty} a_{n}\left\{\left[\sum_{j=1}^{n_{\text {Pan }}}\left(\frac{\partial f}{\partial x}\right)_{j} \Delta P_{n, j}\right]^{2}+\left[\sum_{j=1}^{n_{\text {Pan }}}\left(\frac{\partial f}{\partial x}\right)_{j} \Delta Q_{n, j}\right]^{2}\right\}
$$

Where:

$$
\begin{aligned}
& a_{n}=\frac{2 f_{n} \cosh \left(u_{n}\right)^{2}}{\cosh \left(2 u_{n}\right)} \quad \text { for } \mathrm{n}>0 \\
& f_{n}=2\left[1+\cos \left(\frac{k_{0} S}{2 \sinh \left(2 u_{n}\right)}\right)\right] \\
& \sinh \left(2 u_{n}\right)=\frac{4 \pi n}{k_{0} W} \\
& \left\{\begin{array}{l}
\Delta P_{n, j} \\
\Delta Q_{n, j}
\end{array}\right\}=\iint_{S^{\prime}} e^{z k_{0} \cosh \left(u_{n}\right)^{2}}\left\{\begin{array}{l}
\sin \\
\cos
\end{array}\right\}\left[k_{0} x \cosh \left(u_{n}\right)\right] d S
\end{aligned}
$$

In the above-defined equations the seawater density is denoted as $\rho, S^{\prime}$ represents the projected panel area, $k_{0}=g / U_{\infty}{ }^{2}$ is the wavenumber and $g$ is the gravitational acceleration. The wave-amplitude functions $P$ as well as $Q$ resemble the far field wave system of the demihulls and are expressed analytically in Eq. 6 . The term $f_{n}$ takes the interference of the two demihulls' wave pattern into account and is highly dependent on the demihull spacing $S$, cf. Eq. 4. Kaklis and Papanikolaou (1992) extended the above thin ship theory further to apply to the resistance of twin hull vessels with asymmetric demihulls by assuming normal dipoles in addition to the distribution of Kelvin sources.

The above described approach is numerically implemented in the program RES of the Ship Design Laboratory of NTUA, which calculates $R_{W}$ of thin and slender mono- or twin-hulls. Applications of RES and other numerical methods for the optimisation of various types of twin hull vessels, such as catamarans and SWATHs, are elaborated in Papanikolaou et al. (1996). 


\section{APPLIED METHODOLOGY}

The integration of RES into the CAE system CAESES $^{\circledR}$ as well as the required modifications for the calculation of the catamarans total resistance is accomplished by the herein developed framework cataRES. Since the wave resistance only makes up a moderate fraction of the total resistance in the high-speed regime, the present implemented empirical corrections are mainly addressing the viscous flow effects on the catamaran's resistance, especially in the stern area. According to Insel (1990), the total resistance coefficient $C_{T}$ of catamarans is decomposable in the following way.

$$
C_{T}=C_{F}(1+\beta k)+\tau C_{W}
$$

As stated in the previous section, RES fully accounts for the wave interference resistance expressed in $\tau$. The frictional resistance coefficient $C_{F}$ is calculated according to the ITTC (International Towing Tank Conference) 1957 correlation line.

$$
C_{F}=\frac{0.075}{\left(\log _{10}(R n)-2.0\right)^{2}}
$$

The catamaran's viscous pressure resistance as well as its resistance due to viscous interference $\beta$ are summarised in the catamaran's form factor $(1+\beta k)$. The viscous interference effect refers to the induced resistance due to the circulation around a demihull and the increased frictional resistance by overspeed in the tunnel. The catamaran formfactor is calculated empirically in cataRES as the arithmetic mean of results of the regression formula by Couser et al. (1997) and an interpolated formfactor according to model test data of the NPL round-bilge catamaran hull series (Molland et al. 1994). The first method stems from both model test and full-scale measurements data and is expressed in Eq. 9.

$$
(1+\beta k)=3.03\left(\frac{L}{\nabla}\right)^{-0.4}
$$

The length of the catamaran is denoted as $L$, whereas $\nabla$ represents the displacement of one demihull. The formfactor according to the NPL round-bilge catamaran hull series is linearly interpolated by $L / B$ and $S / L$ as presented in Tab. 1. Since the wave resistance is determined by the wave cut method during the model tests, it complements the potential flow theory calculations by including additional resistances caused by spray and wave-breaking (Molland et al. 1994). The partial formfactor proposed by Dawson (1978) or equivalent techniques, which are commonly applied in traditional Rankine panel methods, seems only reasonable in the lower or medium speed range as an alternative to this empirical approach due to the inherent imperfections of potential flow theory in the high-speed regime.

Table 1: Catamaran formfactors of NPL catamaran round bilge hull series (Molland et al. 1994).

\begin{tabular}{lccccc}
\hline Model & $L / B$ & $S / L=0.2$ & $S / L=0.3$ & $S / L=0.4$ & $S / L=0.5$ \\
\hline 3b & 7.0 & 1.60 & 1.65 & 1.55 & 1.60 \\
$\mathbf{4 a}$ & 10.4 & 1.43 & 1.43 & 1.46 & 1.44 \\
$\mathbf{4 b}$ & 9.0 & 1.47 & 1.43 & 1.45 & 1.45 \\
$\mathbf{4 c}$ & 8.0 & 1.41 & 1.39 & 1.48 & 1.44 \\
$\mathbf{5 a}$ & 12.8 & 1.44 & 1.43 & 1.44 & 1.47 \\
$\mathbf{5 b}$ & 11.0 & 1.41 & 1.45 & 1.40 & 1.38 \\
$\mathbf{5 c}$ & 9.9 & 1.41 & 1.43 & 1.42 & 1.44 \\
$\mathbf{6 a}$ & 15.1 & 1.48 & 1.44 & 1.46 & 1.48 \\
$\mathbf{6 b}$ & 13.1 & 1.42 & 1.40 & 1.47 & 1.44 \\
$\mathbf{6 c}$ & 11.7 & 1.40 & 1.40 & 1.45 & 1.44 \\
\hline
\end{tabular}

Additional implemented modifications to the original thin ship panel method of Papanikolaou and Androulakakis (1991) refer to dynamic trim and sinkage, as well as a transom stern treatment by a virtual appendage. The calculation of dynamic trim and sinkage by use of thin ship theory calculations is in general highly uncertain, Oving (1985). Hence, according to Couser et al. (1998), trim and sinkage were derived from the NPL round-bilge catamaran series data, Molland et al. (1994). However, while the impact of the dynamic trim and sinkage is generally negligible for slender hulls, it may have a considerable impact on transom stern configurations due to the separation of the stern flow particularly in the high-speed regime. Furthermore, the calculation of source densities (cf. Eq. 1) leads to a sink deficit resulting in underestimating the wave resistance. Couser et al. (1998) introduce a method for the transom stern treatment, which considers additional sinks at the transom stern modelled by a virtual appendage, of re-attachment length $H L$. Herein, we adopt the high degree polynomial regression formula of Robards and Doctors (2003), which is based on a comprehensive set of model test data of high-speed displacement vessels, for the determination of $H L$. 


$$
\begin{aligned}
& \frac{H L}{T}=-3.205 \cdot 10^{2} F n_{T}+1.967 \cdot 10^{1} F n_{T}^{2}-4.536 \cdot 10^{2}\left(\frac{B}{T}\right) F n_{T}+7.526 \cdot F n_{T}^{3}+1.094 \cdot 10^{1}\left(\frac{B}{T}\right) F n_{T}^{2}-6.909\left(\frac{B}{T}\right)^{2} F n_{T}- \\
& 3.338 \cdot 10^{-1} F n_{T}^{4}-9.228 \cdot 10^{-3} F n_{T}^{5}+F n_{B}\left[71.33+3.567 F n_{T}+5.49 \cdot 10^{2}\left(\frac{B}{T}\right)-10.1 F n_{T}^{2}+1.712 \cdot 10^{2}\left(\frac{B}{T}\right)^{2}-30.50\right. \\
& \left.+2.054 F n_{T^{-}}^{2} 10.36\left(\frac{\mathrm{B}}{\mathrm{T}}\right)^{3}-1.284\left(\frac{\mathrm{B}}{\mathrm{T}}\right) F n_{T^{2}}^{2} 1.007\left(\frac{\mathrm{B}}{\mathrm{T}}\right)^{2} F n_{T^{-}}-5.559 \cdot 10^{-1} F n_{T}^{4}+6.024 \cdot 10^{-1}\left(\frac{\mathrm{B}}{\mathrm{T}}\right)^{4}+5.438 \cdot 10^{-2} F n_{T}^{5}\right]
\end{aligned}
$$

In Eq. $10, F n_{B}$ is the Froude number referring to the beam and $F n_{T}$ to the draught.

\section{VALIDATION}

The introduced simplified thin ship panel method for the calculation of the resistance of fast catamarans will be validated against model test data and results obtained from a 3D Rankine source panel method as well as a RANSE solver. Both the Wigley hull and the NPL 5 b hull in the $S / L=0.3$ catamaran topology will be assessed. The benchmark for the Wigley catamaran is the EFD (Experimental Fluid Dynamics) data by Insel (1990) and the RANSE computational results by Srinakaew (2017). For the NPL 5b catamaran the basis is the model series data of Molland et al. (1994) and again the CFD results of Srinakaew (2017). Moreover, results of the non-linear 3D panel method code $v$-SHALLO of HSVA are included, noting that it is part of the later employed optimisation procedure, Gatchell et al. (2000).

\subsection{Wigley catamaran}

The half-immersed part of the Wigley hull is discretized by $\sim 500$ triangular and quadrilateral panels for the cataRES computations, whereas the numerical grid for the $v$-SHALLO calculations consists of $\sim 1000$ hull panels and the free surface is discretized additionally according to the default settings. The hull mesh for $v$ SHALLO has to be extended above the stationary waterline, cf. Fig. 2. The wave profile as well as dynamic trim and sinkage are computed iteratively in $v$-SHALLO; thus, the hull grid is subject to change at each iteration. For the sake of brevity grid sensitivity studies are herein not included.

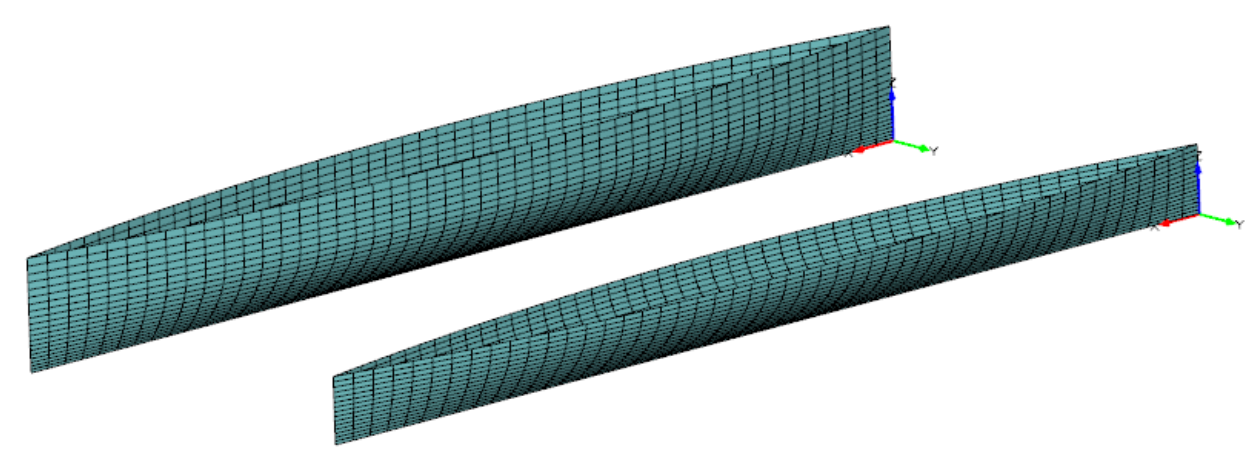

Figure 2: Discretised Wigley demihull for $v$-SHALLO (left) and cataRES (right).

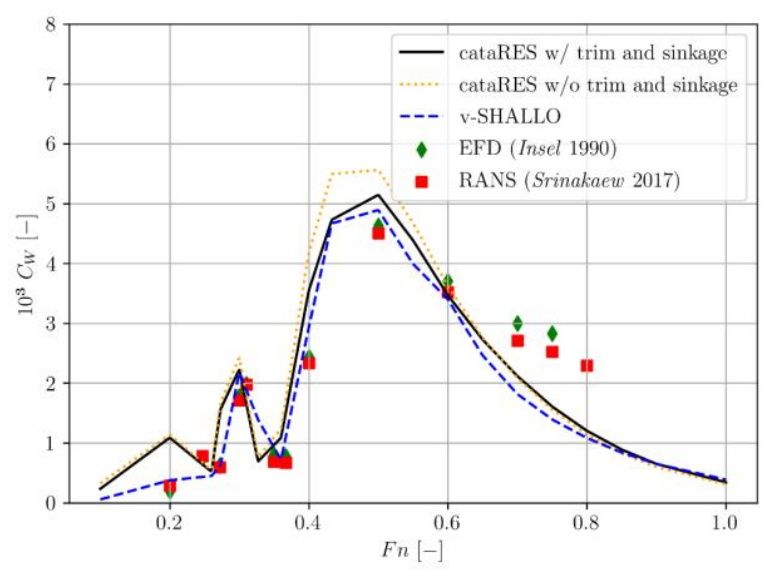

Figure 3: $C_{W}$ for Wigley catamaran $(S / L=0.3)$. 
The agreement between cataRES and $v$-SHALLO in Fig. 3 is particularly remarkable. The significant deviation at $F n=0.2$ stems from the method-typical oscillations of thin ship theory at low speeds (Bertram $1992)$. The comparably low values for cataRES and $v$-SHALLO in high-speed regime $(F n>0.7)$ are attributed to deficiencies of potential flow theory related to stern flow separation at higher speeds, wave breaking and spray phenomena.

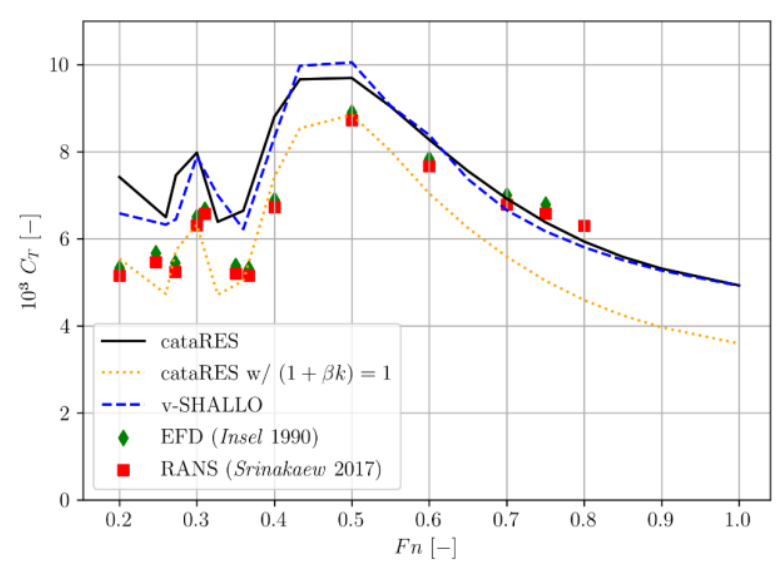

Figure 4: $C_{T}$ for Wigley catamaran $(S / L=0.3)$.

The results of the total resistance coefficient $C_{T}$ in Fig. 4 show that the implemented empirical form factor leads to significantly higher estimates in the range of $0.2 \leq F n \leq 0.5$, whereas in the speed range $0.65 \leq$ $F n \leq 0.8$ - being of interest herein - both panel methods corrected by the empirical viscous form factor yield fully satisfactory results. Obviously, the accuracy of the employed formfactor is highly speed-dependent, while it should be carefully used or even omitted in the low and medium speed range. The ITTC ' 57 correlation line already accounts for minor form effects being seemingly sufficient for slender demihulls in the respective speed range. Of course, more validation studies are necessary for more elaborate conclusions and possible corrections.

\subsection{NPL 5b catamaran}

The half-underwater part of the demihull is discretized by $\sim 500$ panels for the cataRES calculations. The hull grid for $v$-SHALLO comprises includes $\sim 500$ hull panels and the free surface is additionally discretised according to the default settings. The discretised NPL 5 b hull is depicted in Fig. 5 for both panel codes.

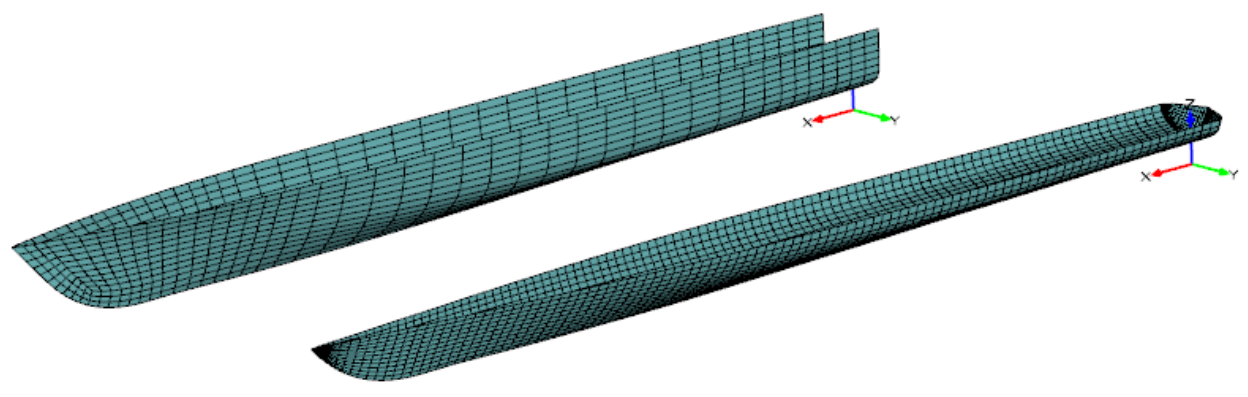

Figure 5: Discretised NPL 5b demihull for $v$-SHALLO (left) and cataRES (right). 


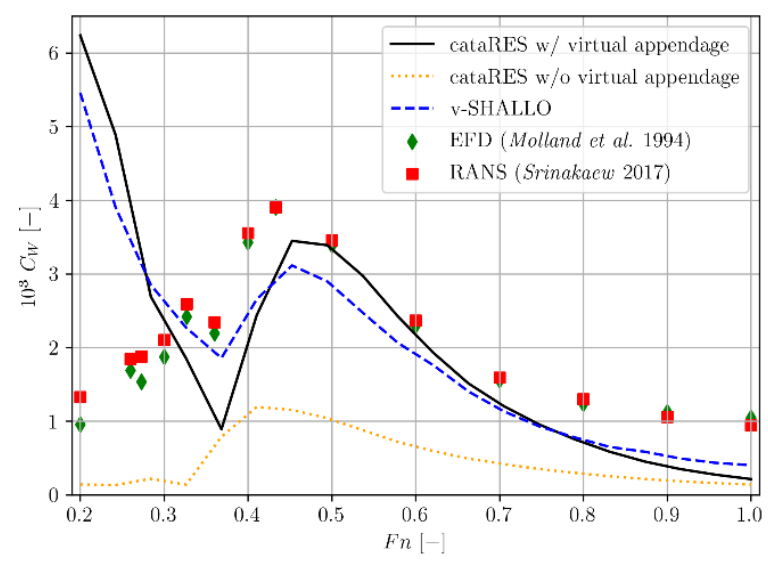

Figure 6: $C_{W}$ for NPL $5 b$ catamaran $(\mathrm{S} / \mathrm{L}=0.3)$.

In view of Fig. 6, the wave resistance coefficient according to cataRES without virtual appendage shows drastic deviations in magnitude in comparison to the benchmark results. Conversely, the addition of the virtual appendage leads, indeed, to a remarkable increase in accuracy for the largest part of the speed range. Considerable deviations are noted, however, in the low speed regime $(0.2 \leq F n \leq 0.32)$ for both panel codes. In the high-speed range $0.7 \leq F n \leq 1.0$, similar discrepancies as in the case of the Wigley hull in Fig. 3 are noted. Thus, it is concluded that the accuracy of the thin ship theory panel method in conjunction with the transom treatment is sufficient for the high-speed range of interest.

\subsection{Concluding Remarks}

The comparisons made with results of resistance for two standard demihull forms obtained from the presented modified thin ship theory panel method, a higher-order 3D panel code, RANSE computations and model tests indicate a satisfactory correlation for the investigated slender hull forms in the high and medium speed range. Furthermore, it shows that a similar degree of accuracy to a Rankine panel method is attainable by the introduced simplified panel code for both mathematical defined and conventional caramaran hull forms. The computing time for the Wigley catamaran discretised by 1,080 panels took slightly more than one second (1.27s) on a workstation with 32 GB RAM, Intel Xeon E3-1231v3 (3.4 GHz) CPU and Linux OS, which is approximately 60 times faster than the higher order panel code $v$-SHALLO. The recommended range of validity of cataRES and the implemented corrections for the application to slender/thin twin hull configurations, as deduced from the herein obtained results, is $0.4 \leq F n \leq 1.0$.

\section{HYDRODYNAMIC HULL FORM OPTIMISATION PROCEDURE}

The hydrodynamic hull form optimisation of the TrAM Stavanger demonstrator is conducted by parallel application of $v$-SHALLO and cataRES. Both codes are integrated into CAESES ${ }^{\circledR}$ using the SoftwareConnector and the open source software package Dakota is used for design exploration and surrogate modelling, Adams et al. (2015). The RANSE solver FreSCo+ (Free Surface Code) of HSVA and TUHH (Hamburg University of Technology) is the benchmark for both panel methods, Hafermann (2007). As part of this optimisation procedure, the calm water resistance of the TrAM catamaran at 21, 23, 25, 27 and 30 knots is defined as objective function, while considering the operational profile; the projected design speed is 23 knots. A multiobjective or Pareto optimisation problem is setup and solved; Eq. 11 shows the minimization of a vector function containing $m$ objective functions.

$$
\min \vec{f}(\vec{x}) \quad \text { where } \vec{f}(\vec{x})=\left[f_{1}(\vec{x}), \ldots, f_{m}(\vec{x})\right]^{T}
$$

The solution vector $\vec{x}$ includes $n$ design variables and is part of the multidimensional design space $\Omega \subseteq \mathbb{R}^{\mathrm{n}}$ Its exploration is carried out with Latin Hypercube Sampling (LHS) and the following optimization - or rather exploitation - is performed using the genetic algorithm NSGA-II. The latter ultimately generates the Paretooptimal or non-dominated designs, which cannot further reduce one objective function without increasing another one. Due to its necessity of multiple iterations, the NSGA-II is applied to surrogate models of the 
objective functions, in order to save computational cost. The final designs are obtained using multi-criteria decision making (MCDM) by utility functions technique, i.e. weighting the individual normalised objective functions. For confidentiality reasons, specific geometric characteristics of the studied catamaran are not included in the presented work and the results are normalised.

\subsection{Parametric Hull Description}

We opted for a fully parametric optimisation approach, because in contrast to the free form deformation method it leads to increased design viability as well as more robust optimisation procedure. Alongside the general challenges of the hydrodynamic optimisation of catamarans, in the case of the TrAM project, a variety of complex requirements and constraints are imposed onto the design process; among others are the requirements for a specific deck platform (module), as well as for a minimum demihull beam, space and weight margin for the fitted batteries and the entire propulsion system - including the electric motor, propeller, rudder and shaft. This is noticeable on one hand in the relatively small variation ranges of the design variables and on the other hand in the constant demihull spacing of the defined parametric hull geometry. The hull model, which was developed by the Ship Design Laboratory of NTUA, is characterised by a round-bilge hull form with a hard chine and a tunnelled stern facilitating the necessary clearance for the fitted large propeller diameters, cf. Fig. 7. The respective design variables are listed in Tab. 2.

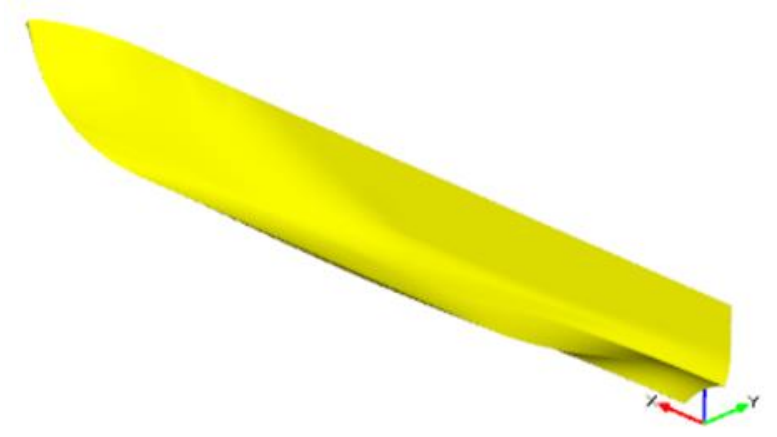

Figure 7: Parametric defined hull description of the Stavanger demonstrator (Kanellopoulou et al. 2019).

Table 2: Design Variables of the parametric hull description

\begin{tabular}{|c|c|c|c|c|}
\hline $\begin{array}{l}\text { Design } \\
\text { Variables } x_{i}\end{array}$ & Description & $\begin{array}{l}\text { Lower } \\
\text { Bound } \\
\end{array}$ & $\begin{array}{l}\text { Upper } \\
\text { Bound }\end{array}$ & Baseline \\
\hline$L W L$ & Length of DWL & 29.0 & 30.6 & 30.1 \\
\hline$H B D E S$ & $\begin{array}{l}\text { Half Beam of } \\
\text { Demihull }\end{array}$ & 1.0 & 1.25 & 1.1 \\
\hline TINIT & Draught & 1.0 & 1.5 & 1.3 \\
\hline$C H I N E \_Y$ & Transom width & 0.8 & 0.85 & 0.81 \\
\hline SHAPE_ATTDES & $\begin{array}{l}\text { Width at DWL } \\
\text { for station } 18\end{array}$ & 0.7 & 1.1 & 0.85 \\
\hline$S H A P E \_L O W$ & $\begin{array}{l}\text { Entrance angle of } \\
\text { station } 18 \text { on CL }\end{array}$ & 0.7 & 1.6 & 1.2 \\
\hline
\end{tabular}

In addition to the transom width (CHINE_Y), the design variables include the main demihull's dimensions (LWL, HBDES, TINIT), whereby the values of the design variables only represent initial values and the values of the actual design waterline are determined using a subroutine implemented into the model ensuring a constant displacement. The optimisation of the fore shoulder profile appears sensible using panel methods due to the small-pronounced boundary layer in the bow region and is performed using the design variables SHAPE_ATTDES and SHAPE_LOW on station 18. A geometrical boundary condition is implemented into the parametric hull description, which assures a minimum installation width for the battery at double bottom height. The described hull model makes up the backbone of the following optimisation procedure and its discretisation for the explorative calculations comprises $\sim 1100$ panels for $v$-SHALLO and $\sim 900$ for cataRES. 


\subsection{Design of Experiments}

A concept exploration or Design of Experiment (DoE) procedure was undertaken to the set problem in order to analyse the basic dependencies of the specified design variables to the objective functions. The datasets were generated by Latin Hypercube Sampling (LHS) and are at a later stage fed into the associated surrogate models. The LHS was proposed by McKay et al. (1979). In contrast to quasi-random sampling methods, LHS carries out stratified sampling for each free variable while taking the entire definition range of them into account by prespecified number of sampling points at a uniform distance. In this context, 200 samples are used for each panel method and speed leading to a set of in total 2,000 calculations. In order to eliminate design variables without considerable influence on the objective functions a correlation analysis is carried out using the Pearson coefficient, defined in Eq. 12.

$$
\mathrm{r}=\frac{\sum_{i=1}^{n}\left(x_{i}-\bar{x}\right)\left(y_{i}-\bar{y}\right)}{\sqrt{\sum_{i=1}^{n}\left(x_{i}-\bar{x}\right)^{2}} \cdot \sqrt{\sum_{i=I}^{n}\left(y_{i}-\bar{y}\right)^{2}}}
$$

The Pearson correlation coefficient is the quotient of the covariance of both variables and of the product of the variables' standard deviations. Noting, that the Pearson coefficient only indicates linear relationships and yields values in the range $-1 \leq r \leq 1$. Here $r=1$ refers to total, positive correlation, for $\mathrm{r}=-1$ it is vice versa and values around zero show no significant relationship. The correlation matrices of the datasets generated by the panel codes are displayed in Tab. 3 .

Table 3: Correlation matrices for RES and $v$-SHALLO

\begin{tabular}{|c|c|c|c|c|c|c|c|c|c|c|}
\hline & \multicolumn{10}{|c|}{ Pearson Correlation Coefficient $r$} \\
\hline & \multicolumn{5}{|c|}{ cataRES } & \multicolumn{5}{|c|}{ v-SHALLO } \\
\hline $\begin{array}{c}\text { Design } \\
\text { Variable }\end{array}$ & $R_{T, 21 k t s}$ & $R_{T, 23 k t s}$ & $R_{T, 25 k t s}$ & $R_{T, 27 k t s}$ & $R_{T, 30 k t s}$ & $R_{T, 21 k t s}$ & $R_{T, 23 k t s}$ & $R_{T, 25 k t s}$ & $R_{T, 27 k t s}$ & $R_{T, 30 k t s}$ \\
\hline \multirow{2}{*}{ LWL } & -0.23 & -0.15 & 0.079 & 0.004 & 0.105 & -0.148 & 0.048 & 0.147 & 0.229 & 0.2926 \\
\hline & - & - & o & o & + & - & o & o & + & + \\
\hline \multirow{2}{*}{ HBDES } & 0.725 & 0.768 & 0.779 & 0.76 & 0.708 & 0.295 & 0.042 & -0.12 & -0.22 & -0.28 \\
\hline & ++ & ++ & ++ & ++ & ++ & + & o & o & - & - \\
\hline \multirow{2}{*}{ TINIT } & 0.107 & 0.103 & 0.092 & 0.0924 & 0.099 & -0.003 & -0.001 & 0.011 & 0.023 & 0.021 \\
\hline & o & o & o & o & o & o & o & o & o & o \\
\hline \multirow{2}{*}{ CHINEY } & -0.158 & -0.155 & -0.124 & -0.072 & 0.0208 & -0.084 & 0.0475 & 0.1717 & 0.236 & 0.27 \\
\hline & - & - & - & o & o & o & o & o & + & + \\
\hline SHAPE_ & -0.42 & -0.381 & -0.378 & -0.408 & -0.467 & -0.65 & -0.723 & -0.725 & -0.698 & 0.668 \\
\hline LOW & - & - & - & - & - & -- & -- & -- & -- & -- \\
\hline SHAPE_ & -0.085 & -0.076 & -0.075 & -0.088 & -0.089 & -0.299 & -0.352 & -0.347 & -0.352 & -0.355 \\
\hline ATTDES & o & $\mathrm{o}$ & o & $\mathrm{o}$ & $\mathrm{o}$ & - & - & - & - & - \\
\hline Note: & $++:$ & strong, & $\Leftrightarrow$ & tion, & & 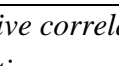 & & & & \\
\hline
\end{tabular}

In both cases, the speed dependence of LWL on the total resistance $R_{T, i}$ is reflected. It further proves that in case of thin ship theory the minimisation of the demihull beam is of paramount importance, which seems trivial in view of Eq. 1. The design variable SHAPE_LOW shifts LCB (Longitudinal Centre of Buoyancy) and its negative correlation shows that the translation of LCB in positive x-direction decreases the total resistance speed-independently. The elimination of TINIT - the draught - would be debatable, yet the correlation in the case of cataRES is quadratic resulting from the formula of the virtual length $H L$, cf. Eq. 10 . In this case, the limitation of the Pearson coefficient in the explorative assessment becomes evident. Ultimately, it is concluded that all examined design variables do have influence on $R_{T, i}$, hence, all of them are taken into account in the following exploitation of the explored first round results.

\subsection{Surrogate Modelling}

Following the DoE procedure, surrogate modelling (or metamodeling) was conducted, which greatly facilitates the optimisation by genetic algorithms by reducing the computational cost significantly. Surrogate modelling is at its core a discipline of supervised machine learning (ML). In the presented work three different types of ML models, suitable for small datasets, are investigated: (1) A non-parametric, Bayesian approach is chosen with Gaussian Process Regression (GPR) - also known as Kriging - like in Harries et al. (2011). (2) 
The instance-based Support Vector Regression (SVR) - see Li et al. (2014) - with a non-linear Radial Basis Function kernel is studied. (3) The stepwise, non-linear regression model MARS (Multivariate Adaptive Regression Splines), which was proposed by Friedman (1991) and is defined in Eq. 13, is also investigated.

$$
\hat{f}(x)=\sum_{m=1}^{M} a_{m}+B_{m}(x)
$$

As inferred in Eq. 13, MARS is a predictive model and the independent variable is a linear combination of base functions $B_{m}$ in a non-parametrical way. The algorithm partitions the feature space into $M$ sub-regions having their own regression equation being determined by forward and backward regression.

Prior to the models' training procedure, the values of the datasets are normalised, since the herein applied algorithms are not scale-invariant. The occurrence of overfitting as well as the models' generalisation capability were assessed by cross validation or out-of-sample testing. The test dataset set makes up $20 \%$ of the initial set and the models are eventually evaluated by the Coefficient of Prognosis $C o P$ (cf. Tab. 4) according to Most and Will (2008). It is stressed that CoP is calculated by squaring Eq. 12 for the test dataset.

Table 4: Comparison of different Surrogate Model architectures

\begin{tabular}{|c|c|c|c|c|c|c|}
\hline & \multicolumn{6}{|c|}{ Coefficient of Prognosis } \\
\hline & \multicolumn{3}{|c|}{ cataRES } & \multicolumn{3}{|c|}{ v-SHALLO } \\
\hline $\begin{array}{c}\text { Function } \\
f i\end{array}$ & GPR & SVR & MARS & GPR & SVR & MARS \\
\hline$R_{T, 2 l k t s}$ & 0.630 & 0.788 & 0.829 & 0.674 & 0.682 & 0.712 \\
\hline$R_{T, 23 k t s}$ & 0.622 & 0.741 & 0.829 & 0.499 & 0.613 & 0.694 \\
\hline$R_{T, 25 k t s}$ & 0.532 & 0.733 & 0.806 & 0.486 & 0.647 & 0.734 \\
\hline$R_{T, 27 k t s}$ & 0.493 & 0.705 & 0.770 & 0.520 & 0.715 & 0.781 \\
\hline$R_{T, 30 k t s}$ & 0.576 & 0.698 & 0.837 & 0.576 & 0.783 & 0.811 \\
\hline Average & 0.571 & 0.733 & 0.814 & 0.551 & 0.688 & 0.746 \\
\hline
\end{tabular}

The MARS algorithm yields higher values of $\mathrm{CoP}$ for all speeds and both panel methods. In the Fig. 8, the accuracy for all algorithms on whole dataset is shown for the theoretical design speed of 23 knots; empty markers represent in-sample data and bounds for relative errors of $\pm 2 \%$ are included.
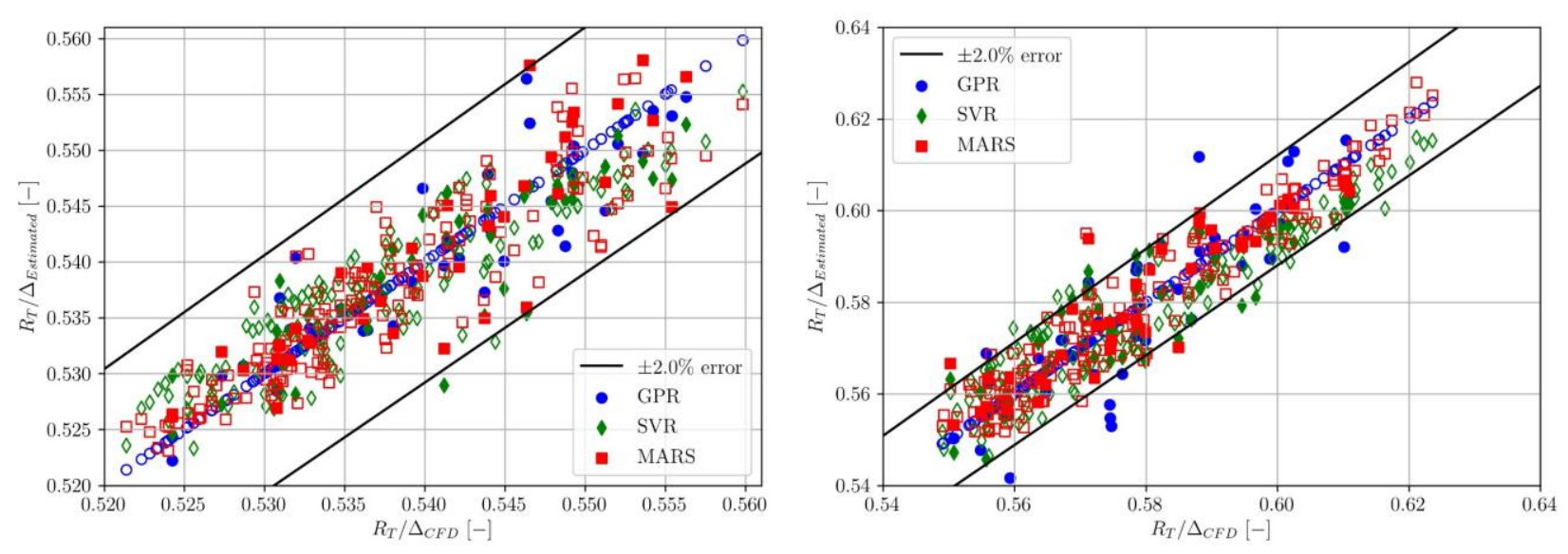

Figure 8: Cross-Validation at $23 \mathrm{kts}$ for cataRES (left) and v-SHALLO (right).

The superior performance of the MARS model is conspicuous once again and the predictions of the linearised thin ship theory are seemingly better to approximate in contrast to the results of the non-linear program $v$-SHALLO. Furthermore, GPR shows great accuracy for in-sample data, but deviant results for the out-of-sample data resulting from the interpolation by a Gaussian process. It shows that this model is overfitted and emphasizes the importance of the applied assessment methodology. As anticipated, the regression model MARS shows superior accuracy and will be used subsequently in the further course of this optimisation procedure. 


\subsection{NSGA-II Exploitation}

The consideration of multiple objective functions requires the use of stochastic or metaheuristic optimisation algorithms - like the genetic algorithms, Nowacki (2003). The underlying principle of a genetic algorithm is the assessment of a population on the basis of a fitness value and the maximization of this quantity by mutation and recombination of the chromosomes - the vector $\vec{x}$ - while keeping a constant population size. The NSGA-II (Non-dominated Sorting Genetic Algorithm) was proposed by Deb et al. (2002) and is a fast, sorting algorithm. The presented exploitation takes 15 generations and a population size of 100 into account. The probability of mutation and recombination are defined as 0.1 and 0.8 , respectively. The optimisation was evaluated by the convergence of hypervolume being calculated by the Pareto-optimal vectors and a reference point. The reference vector consists of doubled $R_{T, i}$ values of the respective baseline design and corresponding scatterplots are presented in the appendix.
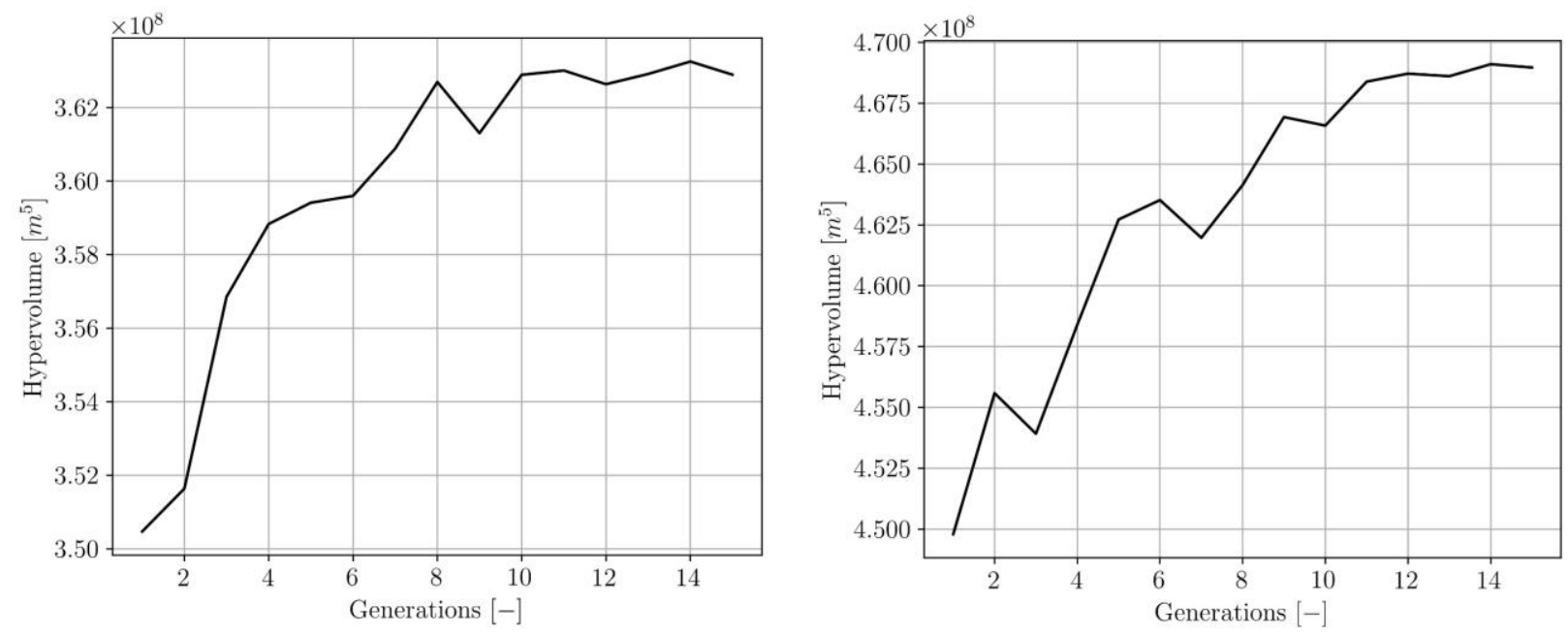

Figure 9: Hypervolume convergence study for cataRES (left) and v-SHALLO (right).

The convergence of hypervolume in the last three generations in Fig. 9 justifies the herein chosen parameters. It is further indicated that the lower magnitude of hypervolume in general and the lower amount of gained hypervolume in case of cataRES implies minor optimisation potential in comparison to $v$-SHALLO. Moreover, it becomes apparent once again, that the minimization of the demihull beam is crucial for cataRES, since the geometric boundary condition is violated significantly more often in comparison to v-SHALLO. Noting, that this is better displayed in the attached scatterplots. These findings lead to the anticipated conclusion that the advanced panel method $v$-SHALLO may be superior to cataRES in context of an optimisation, albeit more demanding in terms of computational effort. Finally, it is thought the assessment of the exploitation by the convergence study of hypervolume offers more insight than scatter plots or parallel coordinate plots; especially in case of a high-dimensional Pareto frontier.

\subsection{Multicriteria Decision Making}

The final designs are ultimately obtained using MCDM by utility functions technique; for greater detail cf. Boulougouris et al. (2004). The weighting of the individual objective functions is the only decisive influence of the user or customer on the optimisation process, which runs almost automatically. The summation of all objective functions is considered as naïve, since this approach neglects the individual orders of magnitude and dimensions of the different objective functions. Hence, a normalised utility function method is applied.

$$
\mathrm{U}=\sum_{\mathrm{i}=1}^{\mathrm{m}}\left\{w_{i} \frac{\max \left(f_{i}\right)-f_{i}}{\max \left(f_{i}\right)-\min \left(f_{i}\right)}\right\}
$$

According to Eq. 14, the normalised objective functions are multiplied by their respective weighting resulting from the preliminary operational profile of the TrAM catamaran, cf. Tab. 5. Note, that $|\vec{w}|=1$ holds. The artificial objective function $U$ yields values in the range $0 \leq U \leq 1$ and $U=1$ corresponds to the ideal design. 
Table 5: Weights for Objective Functions

\begin{tabular}{lccccc}
\hline Objective & $R_{T, 21 k t s}$ & $R_{T, 23 k t s}$ & $R_{T, 25 k t s}$ & $R_{T, 27 k t s}$ & $R_{T, 30 k t s}$ \\
Function $f_{i}$ & 0.2 & 0.5 & 0.15 & 0.1 & 0.05 \\
Weight $w_{i}$ & 0.2 & & & & \\
\hline
\end{tabular}

The theoretical design speed of 23 knots and the enclosing speeds are of paramount importance. The impact is obviously vanishing at higher speeds, since they are probably not attainable in regular service conditions due to the limited battery capacity. The resulting rankings from MCDM of the designs are presented in Fig. 10.
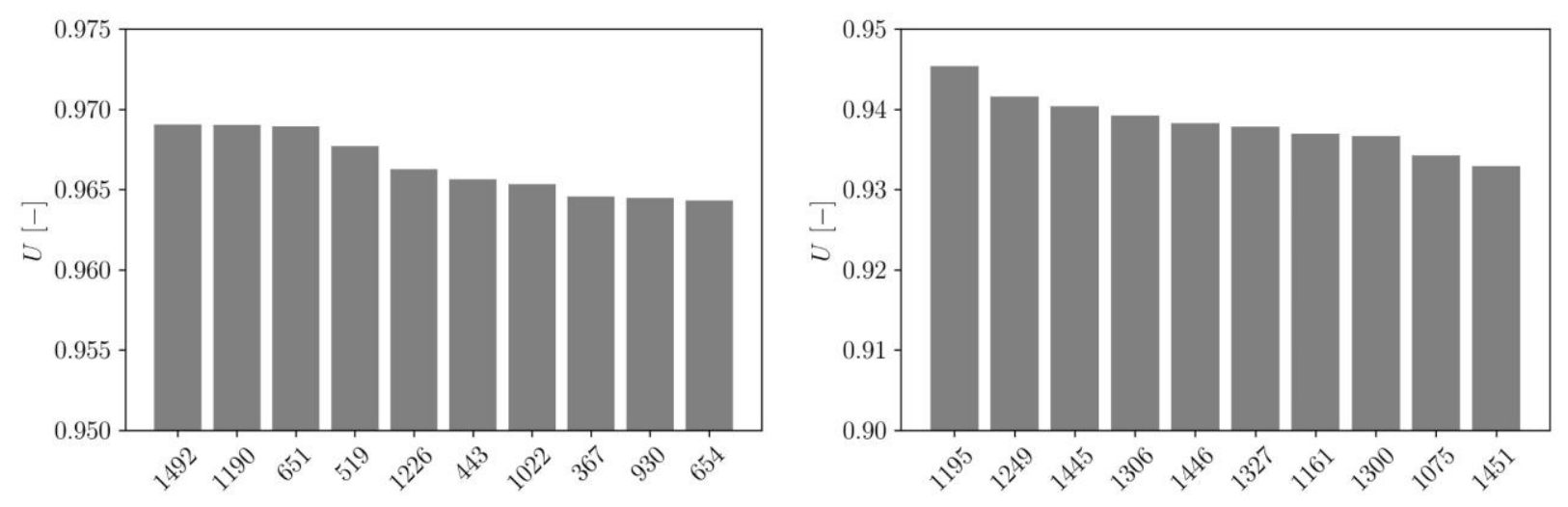

Figure 10: Ranking for cataRES (left) and for $v$-SHALLO (right).

Table 6: Design Variables of the resulting geometries

\begin{tabular}{lccc}
\hline $\begin{array}{l}\text { Design } \\
\text { Variables } x_{i}\end{array}$ & Baseline & RES-1492 & NS-1195 \\
\hline LWL & 30.1 & 30.2227 & 30.4011 \\
HBDES & 1.1 & 1.0637 & 1.0963 \\
TINIT & 1.3 & 1.4878 & 1.3920 \\
CHINE_Y & 0.81 & 0.8318 & 0.8233 \\
SHAPE_ATTDES & 0.85 & 1.0647 & 1.0993 \\
SHAPE_LOW & 1.2 & 1.4629 & 1.4653 \\
\hline
\end{tabular}

In view of Tab 6 and the design variables of RES-1495 and the corresponding ones of the baseline geometry, it is noticeable that the optimal design - according to thin ship theory - is, indeed, characterised by diminished demihull beam leading in turn to an increased draught. The optimal design according to the Rankine panel method NS-1195, on the other hand, shows an increased beam and length, which leads to an enlarged wetted surface area WSA. Regarding the transom width and the shape of the fore shoulder, both variants show similar values emphasizing the capability of the thin ship theory panel method.

\subsection{RANSE-Validation}

Lastly, the three geometries are validated at 23 knots using the finite volume solver FreSCo+ and the RANSE turbulence closure model. Detailed information about the numerical approximation schemes implemented in FreSCo+ are conveyed in Hafermann (2007). The unstructured numerical grid comprises of 2.1 million cells with grid refinements near the hull and the free surface. The $\mathrm{k}-\omega \mathrm{SST}$ turbulence model is used and the free surface elevation is determined by the Volume of Fluid (VoF) method as well as the HighResolution Interface Capturing (HRIC) method. The dimensions of the computational domain comply with the ITTC Guidelines. However, the Froude number leads to an increase in wavelength and to the generation of hydrodynamic lift; hence, the computational domain is elongated (cf. Fig. 11) and trim and sinkage are relaxed considerably. A symmetry boundary condition is enforced on the $0-\mathrm{x}-\mathrm{z}$ plane of the catamaran and a pressure condition is set at the outlet of the domain. The calculations were performed for the full-scale ship without a propeller present, since the focus is on the relative comparison of the three designs instead of accurate power predictions. Results of grid independency studies are again not included herein for the sake of brevity. 


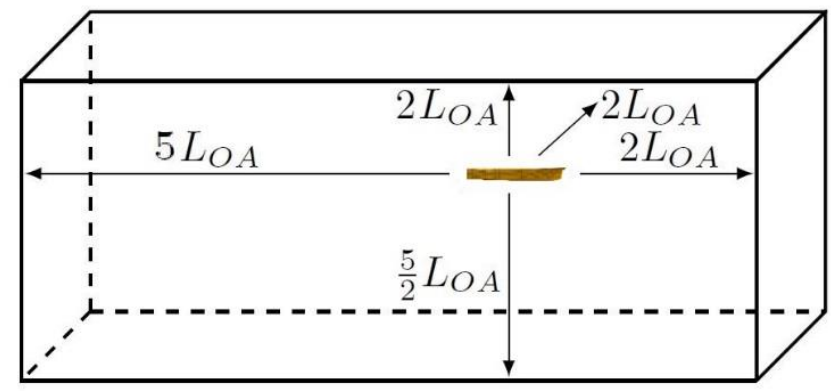

Figure 11: Dimensions of the computational domain.

Table 7: Relative resistance reduction for 23 knots

\begin{tabular}{lccc}
\hline Design & cataRES & v-SHALLO & FreSCo+ \\
\hline NS-1195 & $-1.32 \%$ & $-1.44 \%$ & $+1.2 \%$ \\
RES-1495 & $-1.57 \%$ & $-0.91 \%$ & $-1.1 \%$ \\
\hline
\end{tabular}

Comparing the baseline geometry to RES-1495, it turns out that RES-1495 yields a reduction in total resistance of $1.1 \%$ according to FreSCo+. However, in case of the NS-1195 geometry, it is conspicuous that the total resistance increased by $1.2 \%$. This may be trivial, since the viscous resistance is a function of the dynamic wetted surface area which is increased in case of NS-1195 as pointed out in the previous subsection. Based on these findings, it proves that the thin ship theory panel code is well applicable to the preliminary global optimisation of fast catamarans, leading to the determination of their main dimensions and global form characteristics, in conjunction with the ensuing empirical corrections for the stern flow.

\section{SUMMARY \& CONCLUSIONS}

A simplified panel method based on thin ship theory was modified by empirical corrections, in order to better account for viscous effects and the stern flow of fast catamarans. The implemented numerical method was systematically validated and applied to the parametric multi-objective optimisation of a fast battery-driven catamaran. The obtained results show satisfactory applicability of the employed semi-empirical calculation method in the realm of the preliminary design of fast catamarans, while the computational cost is greatly reduced in comparison with higher-order panel methods and RANSE codes.

The herein presented optimisation of the calm water resistance may be enhanced by additional objectives, e.g. the seakeeping attitude and even added-wave resistance. Moreover, the occurrence of wave wash is a wellknown problem of fast displacement catamarans, hence an optimisation procedure minimising a wash criterion as shown by Zaraphonitis et al. (2003) seems reasonable. Thus, it may be promising to extend the presented code for calculations in finite water depth, e.g. by considering of the work of Eggers (1955), thus, better accounting for the wave wash effect in shallow waters.

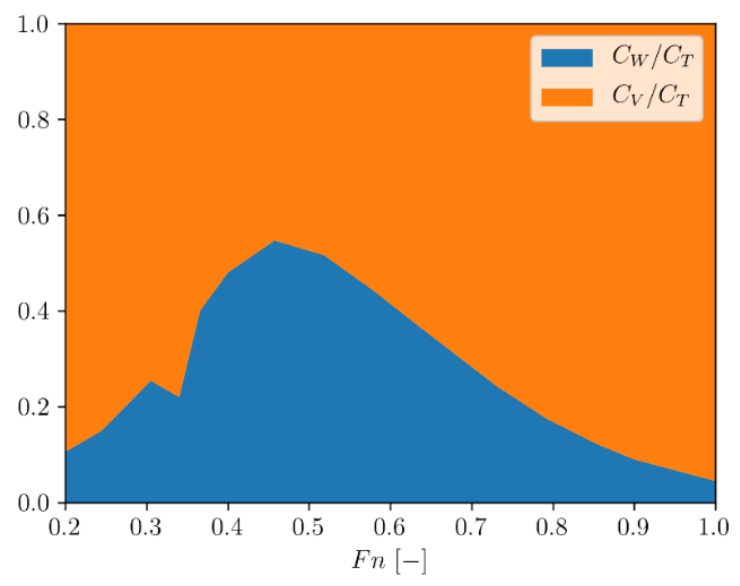

Figure 12: Wave-resistance fraction for the TrAM baseline geometry according to cataRES. 
The wave resistance of fast catamarans is, in fact, only a small fraction of the total resistance $\left(\sim 0.25 C_{T}\right)$ in the high-speed regime, cf. Fig. 12. Nonetheless, the wave resistance is a decisive factor for the determination of the catamaran's main dimensions and form characteristics in the frame of a global optimisation. Conversely, for the local hull form optimisation, the design exploration needs to be based on a viscous flow solver - like FreSCo+. This includes the optimisation of the catamaran's propulsive performance, i.e. the optimisation of stern tunnel area, propeller and appendages, as shown in related works of the TrAM project (TrAM 20182022). When using a RANSE solver anyhow, an optimisation approach by an adjoint VoF method - like proposed by Kröger et al. (2016) - for fast catamarans shows great potential for future work.

Resistance and propulsion model tests of the Stavanger demonstrator were carried out at HSVA in December of 2019 and repeated in May 2020; obtained results generally confirmed the validity of the herein applied resistance calculation methods and optimisation procedures. The construction of the Stavanger prototype is scheduled to start in early 2021 and to enter service in the first half of 2022 (TrAM 2018-2022). The project will also carry out two studies for the operation of similar zero-emission passenger vessels in London/Thames River and Belgium.

\section{ACKNOWLEDGEMENTS}

The presented work is the core part of the Master thesis of the $1^{\text {st }}$ author at the Univ. of Applied Sciences, Kiel supervised by Professor Graf. The thesis was conducted at the CFD Department of HSVA under the supervision and guidance of the $2^{\text {nd }}$ author. The valuable support given by the staff of HSVA and the Ship Design Laboratory of NTUA is greatly acknowledged. The referenced projects TrAM (No. 769303) and HOLISHIP (No. 689074) have received funding from the European Union's Horizon 2020 research and innovation programme.

\section{REFERENCES}

Adams, B.M., Bauman, L.E., Bohnhoff, W.J., Dalbey, K.R., Ebeida, M.S., Eddy, J.P., Eldred, M.S., Hough, P.D., Hu, K.T., Jakeman, J.D., Stephens, J.A., Swiler, L.P., Vigil, D.M., and Wildey, T.M. (2015) Dakota, A Multilevel Parallel Object-Oriented Framework for Design Optimization, Parameter Estimation, Uncertainty Quantification, and Sensitivity Analysis: Version 6.0 User's Manual. Sandia Technical Report SAND2014-4633

Bertram, V.; Krebber, B.; Hochkirch, K.; Frers, G.; Flynn, M. (2017) Advanced simulation-based design for an advanced power trimaran. Proc. $11^{\text {th }}$ Symp. on High Speed Marine Vehicles (HSMV), Naples

Bertram, V. (2017) Ship Design Technologies - A CAVE Man's View. Proc. $11^{\text {th }}$ Symp. on High-Performance Marine Vehicles (HIPER'17), Zevenwacht

Bertram, V. (1992) Wave Resistance Calculation for SWATH Ships and Catamarans. (in German) Jahrbuch der Schiffbautechnischen Gesellschaft Vol. 86, Springer

Boulougouris, E.; Papanikolaou, A.; Zaraphonitis, G. (2004) Optimization of Arrangements of Ro-Ro Passenger Ships with Genetic Algorithms. Ship Tech. Res., Vol. 51, No. 3, pp 99-105

Chapman, R.B. (1972) Hydrodynamic drag of semi-submerged ships. ASME, J. Basic Eng., Vol. 94 No. 4, pp. 879-84

Couser, P.; Molland, A.; Armstrong, N.; Utama, I. (1997) Calm Water Powering-Prediction for High-Speed Catamarans. Proc. Int. Conf. on Fast Sea Transportation (FAST '97), Sydney, Australia

Couser, P.; Wellicome, J.; Molland, A. (1998) An Improved Method for the Theoretical Prediction of the Wave Resistance of Transom-Stern Hulls Using a Slender Body Approach. International Shipbuilding Progress, Vol. 45, No. 444, pp 331-349

Danisman, D.B.; Mesbahi, E.; Atlar, M.; Gören, Ö. (2002) A New Hull Form Optimization Technique for Minimum Wave Resistance. Proc. $10^{\text {th }}$ Int. Maritime Association of the Mediterranean Congress I.M.A.M., Crete

Dawson, C.W. (1978) Calculations with the xyz Free Surface program for five ship models. Proc. Workshop on Ship Wave Resistance Computations, DTNSRDC, Bethesda, pp. 232-255

Deb, K.; Pratap, A.; Agarwal, S.; Meyarivan, T. (2002) Fast and Elitist Multiobjective Genetic Algorithm: NSGA-II. IEEE Transactions on Evolutionary Computation, Vol. 6, No. 2, pp 182-197

Eggers, K. (1955) Resistance components of two-body ships. (in German) Jahrbuch der Schiffbautechnischen Gesellschaft Vol. 49, pp 516-539 
Faltinsen, O.M. (2006) Hydrodynamics of High-Speed Marine Vehicles. Cambridge University Press, ISBN 9780521178730

Friedman, J. (1991) Estimating Functions of Mixed Ordinal and Categorical Variables Using Adaptive Splines, Technical Report No. 108 - Laboratory for Computational Statistics, Department of Statistics Stanford University.

Gatchell, S.; Hafermann, D.; Jensen, G.; Marzi, J.; Vogt, M. (2000) Wave resistance computations - A comparison of different approaches, Proc. $23^{\text {rd }}$ Symp. on Naval Hydrodyn. (ONR), Val de Reuil

Hafermann, D. (2007) The New RANSE Code FreSCo for Ship Applications. Proc. of Annual Meeting Schiffbautechnische Gesellschaft, STG Jahrbuch

Harries, S.; Abt, C. (2019) The HOLISHIP Platform for Process Integration and Design Optimization. A Holistic Approach to Ship Design, Volume 1: Optimisation of Ship Design and Operation for Life Cycle. Springer 978-3-03002809-1, pp. 247-293

Harries, S.; Tillig, F.; Wilken, M.; Zaraphonitis, G. (2011) An integrated approach for simulation in the early ship design of a tanker. Proc. $10^{\text {th }}$ Int. Conf. on Computer and IT Applications in the Maritime Industries (COMPIT), Berlin, pp. 206-220

Hess, J.L.; Smith, A.M.O. (1967) Calculation of potential flow about arbitrary bodies. Progress in Aerospace Science, Vol. 1, pp. 1-138

HOLISHIP Project, 2016-2020 HOLISHIP (HOLIstic optimisation of SHIP design and operation for life cycle). EU funded Horizon 2020 project, https://www.holiship.eu/

Insel, M. (1990) An investigation into Resistance Components of High-Speed Displacement Catamarans, Ph.D. thesis, Department of Ship Science, University of Southampton

Kaklis, P.D.; Papanikolaou, A.D. (1992) The Wave Resistance of a Catamaran with Non-Symmetric Thin Hulls, Proc. $1^{\text {st }}$ Nat. Congress on Computational Mechanics, (GRACM), Athens

Kanellopoulou, A.; Xing-Kaeding, Y.; Mittendorf, M. (2019) Parametric Design and Optimization of a Battery-driven Catamaran in CAESES. CAESES Users Meeting, Berlin

Kröger, J.; Kühl, N.; Rung, T. (2018) Adjoint volume-of-fluid approaches for the hydrodynamic optimisation of ships, Ship Tech. Res., Vol. 65, No. 1, pp. 47-68

Lagally, M. (1922) Calculation of forces and moments acting by stream flows on their boundaries. (in German) Zeitschrift f. Angewandte Mathematik und Mechanik (ZAMM), Vol. 2, No. 6, https://doi.org/10.1002/zamm.19220020601

Li, D.; Guan, Y.; Wilson, P.; Zhao, X. (2014) An Effective Approximation Modeling Method for Ship Resistance in Multidisciplinary Ship Design Optimization. Proc. $33^{\text {rd }}$ Int. Conf. on Ocean, Offshore and Arctic Eng. OMAE 2014, San Francisco

McKay, M.D.; Beckman, R.J.; Conover, W.J. (1979) A comparison of three methods for selecting values of input variables in the analysis of output from a computer code, Technometrics, Vol. 21 No. 2, pp. 239-245

Michell, J.H. (1898), The wave resistance of a ship, Phil. Mag. Vol. 45, London, pp. 106-123

Mittendorf, M. (2019) Development and Application of a Resistance Optimisation Procedure for Catamarans with the Design Software CAESES. (in German) Master thesis at Univ. of Applied Sciences Kiel

Molland, A.; Wellicome, J.; Couser, P. (1994) Resistance experiments on a systematic series of high-speed displacement catamaran forms: Variation of length-displacement ratio and breadth-draught ratio. Ship Science Report No. 71, Department of Ship Science, University of Southampton

Most, T.; Will, J. (2008) Metamodel of Optimal Prognosis - An automatic approach for variable reduction and optimal metamodel selection. Proc. Weimar Optimization and Stochastic Days 2008, Weimar

Nowacki, H. (2003) Design Synthesis and Optimization - An Historical Perspective. OPTIMISTIC - Optimization in Marine Design, 39 ${ }^{\text {th }}$ WEGEMT Summer School, Berlin

Oving, A. (1985) Resistance Prediction Method for Semi-Planing Catamarans with symmetrical Demihulls. Diploma thesis at TH Delft, Wageningen

Papanikolaou, A.D. (2010) Holistic Ship Design Optimization. Journal Computer-Aided Design, Elsevier, Vol. 42 Issue 11 , pp. $1028-1044$

Papanikolaou, A.; Androulakakis, M. (1991) Hydrodynamic Optimization of High-Speed SWATH. Proc. $1^{\text {st } I n t . ~ C o n f . ~}$ on Fast Sea Transportation (FAST '91), Trondheim 
Papanikolaou, A., Kaklis, P., Koskinas, C., Spanos, D. (1996) Hydrodynamic Optimization of Fast Displacement Catamarans", Proc. 21 ${ }^{\text {st }}$ Int. Symposium on Naval Hydrodynamics, ONR' 96, Trondheim

Robards, S.; Doctors, L. (2003) Transom Hollow Prediction for High-Speed Displacement Vessels. Proc. $7^{\text {th }}$ Int. Conf. on Fast Sea Transportation (FAST '03), Vol. 1 pp 19-26, Ischia

Srinakaew, S. (2017) A numerical study of resistance components of high-speed catamarans and the scale effects on form factor, Ph.D. thesis at University of Southampton, Faculty of Engineering and the Environment

Srettensky, L.N. (1936) On the wave-making resistance of a ship moving in a canal. Phil. Mag. Vol. 22, pp. 1005

TrAM Project, 2018-2022. Transport: Advanced and Modular, EU funded Horizon 2020 project, https://tramproject.eu

Zaraphonitis, G.; Papanikolaou, A.; Mourkoyannis, D. (2003) Hull form optimization of high-speed vessels with respect to wash and powering. Proc. $8^{\text {th }}$ Int. Marine Design Conference (IMDC), Vol. 3, pp 43-54, Athens

\section{APPENDIX}

\section{NSGA-II EXPLOITATION PLOTS}
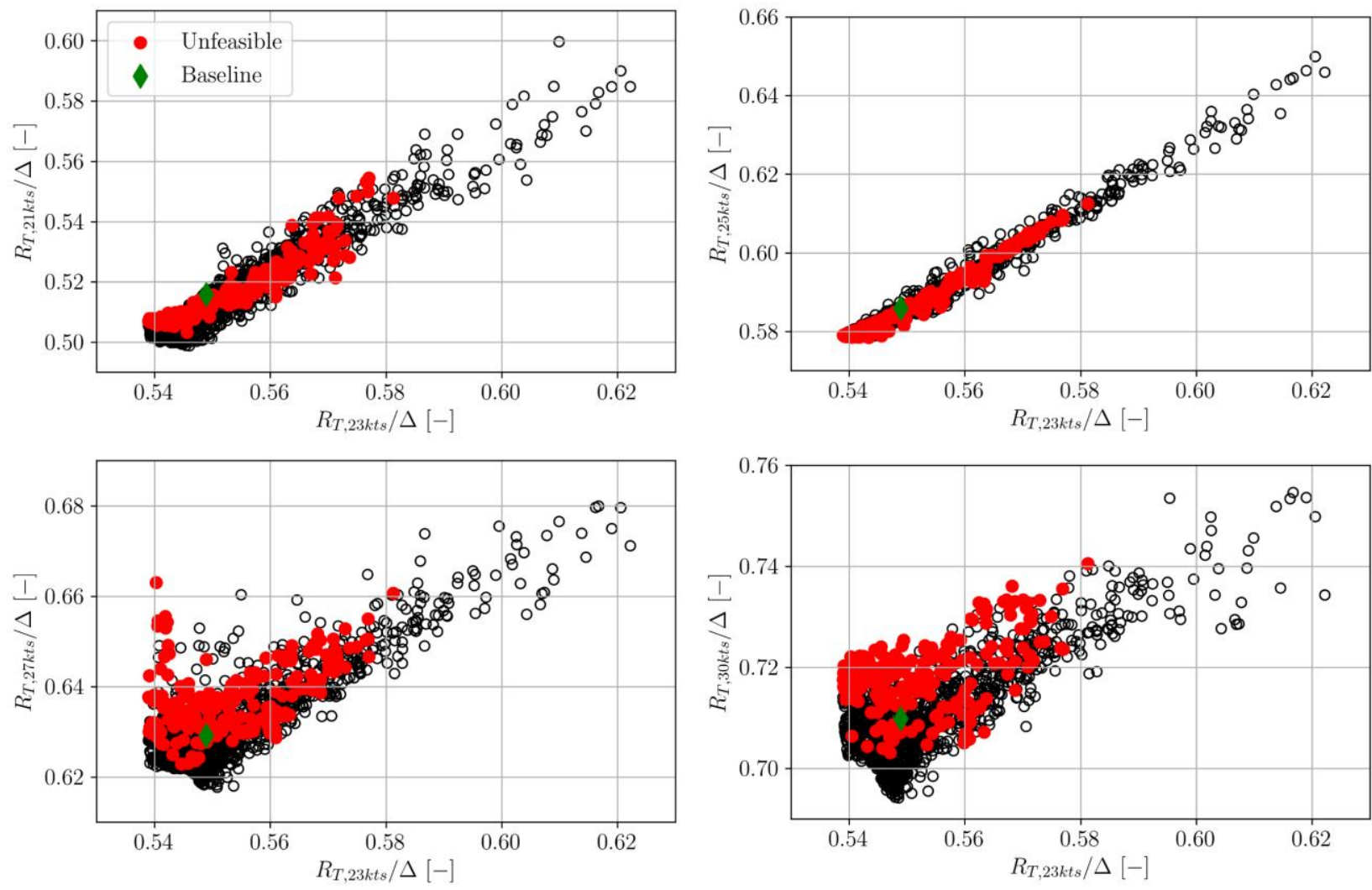

NSGA-II Exploitation for cataRES 

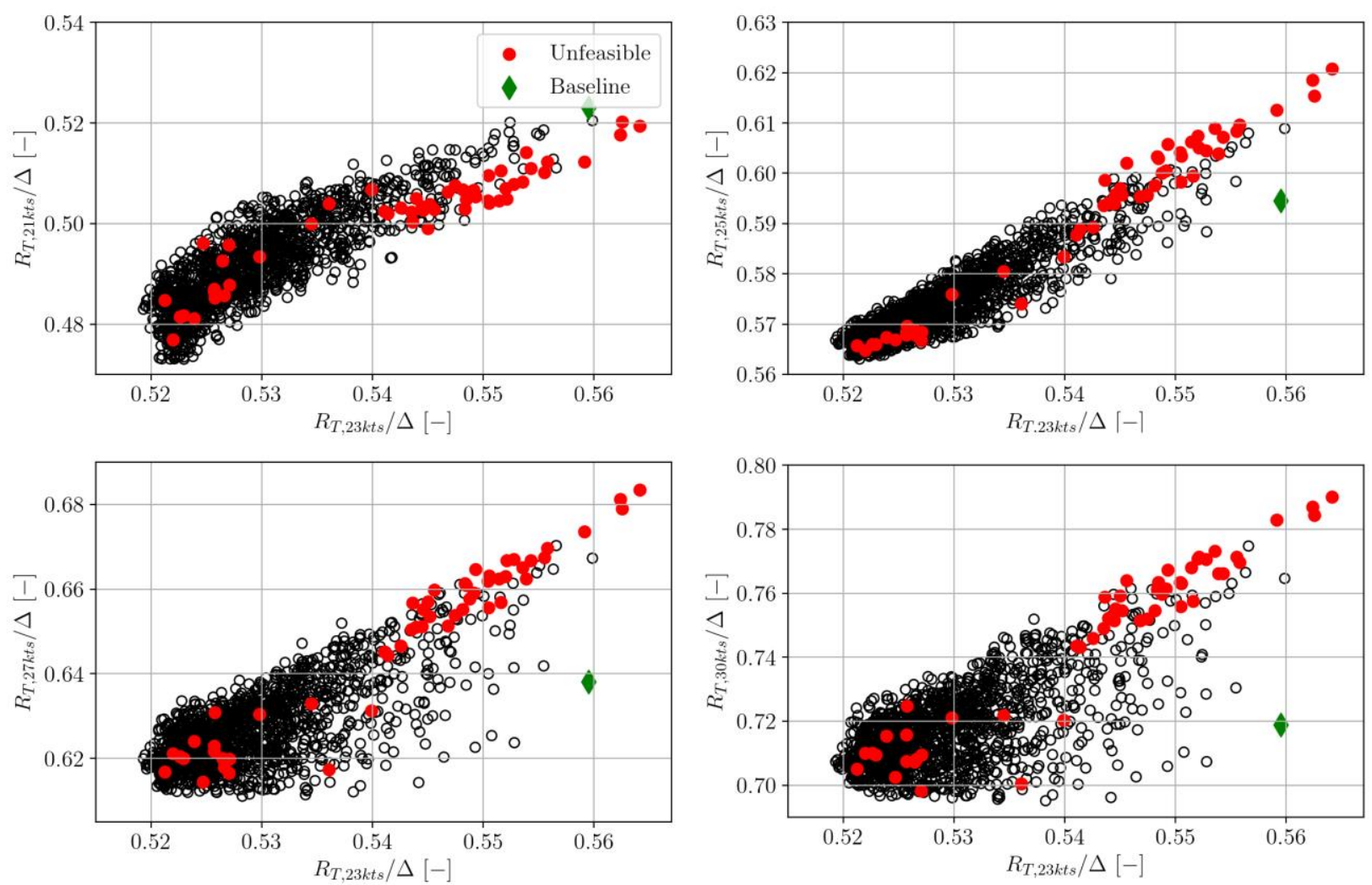

NSGA-II Exploitation for $v$-SHALLO 